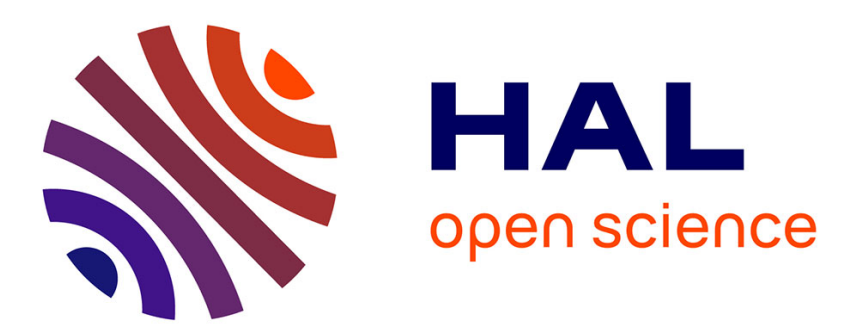

\title{
Long time accuracy of Lie-Trotter splitting methods for Langevin dynamics
}

Assyr Abdulle, Gilles Vilmart, Konstantinos Zygalakis

\section{To cite this version:}

Assyr Abdulle, Gilles Vilmart, Konstantinos Zygalakis. Long time accuracy of Lie-Trotter splitting methods for Langevin dynamics. SIAM Journal on Numerical Analysis, 2015, 53 (1), pp.1-16. 10.1137/140962644. hal-00965354v2

\section{HAL Id: hal-00965354 https://hal.science/hal-00965354v2}

Submitted on 11 Sep 2014

HAL is a multi-disciplinary open access archive for the deposit and dissemination of scientific research documents, whether they are published or not. The documents may come from teaching and research institutions in France or abroad, or from public or private research centers.
L'archive ouverte pluridisciplinaire HAL, est destinée au dépôt et à la diffusion de documents scientifiques de niveau recherche, publiés ou non, émanant des établissements d'enseignement et de recherche français ou étrangers, des laboratoires publics ou privés. 


\title{
Long time accuracy of Lie-Trotter splitting methods for Langevin dynamics
}

\author{
Assyr Abdulle ${ }^{1}$, Gilles Vilmart ${ }^{2}$, and Konstantinos C. Zygalakis ${ }^{3}$
}

September 9, 2014

\begin{abstract}
A new characterization of sufficient conditions for the Lie-Trotter splitting to capture the numerical invariant measure of nonlinear ergodic Langevin dynamics up to an arbitrary order is discussed. Our characterization relies on backward error analysis and needs weaker assumptions than assumed so far in the literature. In particular, neither high weak order of the splitting scheme nor symplecticity are necessary to achieve high order approximation of the invariant measure of the Langevin dynamics. Numerical experiments confirm our theoretical findings.

Key words. stochastic differential equations, splitting method, Langevin dynamics, weak convergence, modified differential equations, backward error analysis, invariant measure, ergodicity.
\end{abstract}

AMS subject classifications. 65C30, 60H35, 37M25.

\section{Introduction}

In many applications (see $[25,16,5,19]$ and references therein), one is interested in simulating the invariant measure of a stochastic differential equation (SDE) by running a numerical scheme that approximates its time dynamics. In particular, one uses the numerical trajectories to construct an empirical measure either by averaging one single long trajectory or by averaging over many realisations to obtain a finite ensemble average (see for example [25]). One immediately is faced with the question regarding the quality of such an approximation. Another issue is the ergodicity of the numerical approximation itself. This second question is still an area of active research [20,33, 28, 31], but is not addressed here.

In this paper, we focus on the case of second order stochastic dynamics generated by the Langevin equation, of the form

$$
\begin{aligned}
d q(t) & =M^{-1} p(t) d t \\
d p(t) & =(-\nabla V(q(t))-\gamma p(t)) d t+\sqrt{2 \beta^{-1} \gamma} M^{1 / 2} d W(t),
\end{aligned}
$$

\footnotetext{
${ }^{1}$ Mathematics Section, École Polytechnique Fédérale de Lausanne, Station 8, 1015 Lausanne, Switzerland, Assyr.Abdulle@epfl.ch

${ }^{2}$ Université de Genève, Section de mathématiques, 2-4 rue du Lièvre, CP 64, 1211 Genève 4, Switzerland, Gilles.Vilmart@unige.ch

${ }^{3}$ Mathematical Sciences, University of Southampton, Southampton SO17 1BJ, UK, k.zygalakis@soton.ac.uk
} 
where the scalar function $V: \mathbb{R}^{d} \rightarrow \mathbb{R}$ denotes the potential, the mass matrix $M$ is symmetric positive definite, $\gamma>0$ (the friction), $\beta>0$ (related to the temperature) are fixed constants, $p(t), q(t) \in \mathbb{R}^{d}$, and $W(t)$ denotes a standard $d$-dimensional Wiener process. For simplicity, we assume that the mass matrix is the identity matrix $M=I$, but we emphasize that our analysis would apply straightforwardly to general positive definite matrices $M$. Under appropriate smoothness and growth assumptions on the Hamiltonian energy (see e.g. [21, 14]),

$$
H(p, q)=\frac{1}{2} p^{T} p+V(q),
$$

one can show that the dynamics generated by (1) are ergodic, i.e. for arbitrary initial conditions $q(0), p(0)$ (assumed deterministic for simplicity), and for all smooth test function $\phi \in \mathcal{C}_{P}^{\infty}\left(\mathbb{R}^{2 d}, \mathbb{R}\right)$ with derivatives of all orders with polynomial growth, the time average of the trajectories of (1) satisfy with probability 1 ,

$$
\lim _{T \rightarrow \infty} \frac{1}{T} \int_{0}^{T} \phi(p(t), q(t)) d t=\int_{\mathbb{R}^{d} \times \mathbb{R}^{d}} \phi(p, q) d \mu(p, q) .
$$

The invariant measure $\mu$ is characterized by the Gibbs density function

$$
\rho_{\infty}(p, q)=Z e^{-\beta H(p, q)},
$$

where $Z$ is the normalization constant, which ensures that $\int_{\mathbb{R}^{d} \times \mathbb{R}^{d}} \rho_{\infty}(p, q) d p d q=1$. Similarly to [4], we consider the following class of schemes based on the Lie-Trotter splitting,

$$
X_{n+1}=\Phi_{h} \circ \Theta_{h, n}\left(X_{n}\right),
$$

where $X_{n}=\left(p_{n}, q_{n}\right)^{T}$. The integrator $\Phi_{h}$ approximates the exact flow with time $h$ of the deterministic Hamiltonian part

$$
d q(t)=p(t) d t, \quad d p(t)=-\nabla V(q(t)) d t,
$$

while $\Theta_{h, n}$ is a numerical integrator for the stochastic part

$$
\begin{aligned}
& d q(t)=0, \\
& d p(t)=-\gamma p(t) d t+\sqrt{2 \beta^{-1} \gamma} d W(t) .
\end{aligned}
$$

The exact solution of (6) is given by the variation of constants formula,

$$
q\left(t_{n+1}\right)=q\left(t_{n}\right), \quad p\left(t_{n+1}\right)=e^{-\gamma h} p\left(t_{n}\right)+\sqrt{2 \beta^{-1} \gamma} \int_{t_{n}}^{t_{n+1}} e^{-\gamma(h-s)} d W(s) .
$$

The trajectories of the above stochastic integral itself need not be simulated. Indeed, the properties of the stochastic integral [26] imply that the solution $p(t)$ of $(6)$ given $p(0)$ is Gaussian and thus characterized by its mean and covariance. Using this, we obtain the following flow $\left(q_{n+1}, p_{n+1}\right)=\Theta_{h, n}\left(q_{n}, p_{n}\right)$ for (6) which is exact in law, ${ }^{1}$

$$
q_{n+1}=q_{n}, \quad p_{n+1}=e^{-\gamma h} p_{n}+\sqrt{\beta^{-1}\left(1-e^{-2 \gamma h}\right)} \xi_{n}
$$

\footnotetext{
${ }^{1}$ This means that the exact solution $(p(h), q(h))$ and the numerical solution $\Theta_{h, n}(p(0), q(0))$ have the same law of probability for a given state $(p(0), q(0))$.
} 
where $\xi_{n} \sim \mathcal{N}(0, I)$ are independent $d$-dimensional Gaussian random variables.

The aim of this paper is to analyse splitting schemes such as (4) for a variety of different deterministic integrators $\Phi_{h}$ used for solving (5), for the numerical approximation of the invariant measure, i.e. we quantify the error

$$
e(\phi, h):=\lim _{N \rightarrow \infty} \frac{1}{N} \sum_{n=1}^{N} \phi\left(p_{n}, q_{n}\right)-\int_{\mathbb{R}^{d} \times \mathbb{R}^{d}} \phi(p, q) \rho_{\infty}(p, q) d p d q,
$$

for all smooth test function $\phi \in \mathcal{C}_{P}^{\infty}\left(\mathbb{R}^{2 d}, \mathbb{R}\right)$. We say that the method has order $r$ for sampling the invariant measure if $|e(\phi, h)| \leq C h^{r}$ for all $h$ small enough, where $C$ is independent of $h$, but depends on the smooth test function $\phi$. In particular, using recent advances in the theory of modified integrators for SDEs and ODEs $[2,8]$ and backward error analysis for SDEs [35, 9, 14, 15, 3], we provide a new characterization of sufficient conditions for the Lie-Trotter splitting to capture the numerical invariant measure of nonlinear ergodic Langevin dynamics up to an arbitrary order.

It is well known that controlling the weak error $\mid \mathbb{E}\left(\phi\left(X_{n}\right)\right)-\mathbb{E}\left(\phi\left(X\left(t_{n}\right)\right) \mid\right.$ up to a given order allows to control the accuracy error $|e(\phi, h)|$ for the invariant measure up to the same order [34],[22]. However, the converse is not true, and new sufficient conditions independent of the weak order of accuracy for a numerical method to approximate with high order of accuracy the invariant measure of ergodic SDEs have recently been derived in [3]. The accuracy of the numerical computation of the invariant measure for Langevin dynamics has recently been studied in a number of papers $[4,16,17]$. In [4], the splitting method (4) is proposed and analyzed, while several other splitting methods with high order for the invariant measure are considered and studied in $[16,17]$. It is shown in [4] that if $\Phi_{h}$ is a variational integrator (equivalently a symplectic scheme) which preserves the energy up to order $r$, then the Lie-Trotter splitting (4) has order $r$ of accuracy for the invariant measure, although the standard weak error is only of order one. This is another example illustrating the fact that a high weak order of accuracy is not necessary to achieve high order for the invariant measure.

In this paper, we provide weaker assumptions to achieve order $r$ in the approximation of the numerical invariant measure using the splitting (4). Our results show that to achieve order $r$ for the invariant measure by the splitting (4), neither an order $r$ of accuracy in the weak sense nor an order $r$ of accuracy in the deterministic sense for $\Phi_{h}$ in (4) are needed. This is also related to the results in $[6,7]$ which provide a detailed analysis of the accuracy of the numerical invariant measure in the case of a linear drift, i.e. a quadratic potential $V(q)$. It is shown there that among the class of stochastic Runge-Kutta methods applied to Langevin dynamics with a linear drift (which does not include the Lie-Trotter splitting (4)), the (stochastic) implicit midpoint rule is the only (stochastic) Runge-Kutta method with a nonsingular tableau matrix that reproduces the exact stationary distribution for all values of damping. See also related results in [19]. Our conclusion for Lie-Trotter splitting methods (4) is different: we prove in particular that it samples exactly the invariant measure for a linear drift if the deterministic integrator $\Phi_{h}$ is a consistent symmetric Runge-Kutta method.

Our new characterization is expressed in terms of functions arising in the modified differential equation for the Hamiltonian system. We thus heavily rely on backward error analysis for our finding. The first error term in the error estimate for the numerical invariant measure can also be expressed in term of functions arising in the modified differential equation. This is also a new expression for error estimates à la Talay-Tubaro [34]. 
To simplify the presentation of our results, we focus on the globally Lipschitz case and we assume that the potential $V$ is a $\mathcal{C}^{\infty}$ function where $\nabla V$ has bounded derivatives of any order (which implies the global Lipschitzness of $\nabla V$ ) and satisfies the following standard growth condition, which guaranties that (1) is ergodic,

$$
q^{T} \nabla V(q) \geq C_{1} q^{T} q-C_{2}, \quad \text { for all } q \in \mathbb{R}^{d},
$$

for some constants $C_{1}, C_{2}>0$. We further assume that the numerical flow $\Phi_{h}$ is globally Lipschitz in $\mathbb{R}^{2 d}$,

$$
\left\|\Phi_{h}\left(x_{1}\right)-\Phi_{h}\left(x_{2}\right)\right\| \leq L\left\|x_{1}-x_{2}\right\|
$$

for all $x_{1}, x_{2} \in \mathbb{R}^{2 d}$ and all stepsize $h$ small enough. In a more general non-globally Lipschitz setting, important in most applications, two implicit schemes are studied in [14], and the implicitness is used to guarantee the boundedness of the numerical moments along time. Using implicit schemes for the Hamiltonian part is one way to extend our results in the non-globally Lipschitz setting. Alternatively, notice that the class of integrators considered in this paper (which are not assumed implicit) could still be applied rigorously in the non-globally Lipschitz setting by following the methodology of rejecting exploding trajectories proposed in [24] and applied to Langevin-type ergodic systems in [25].

We close this introduction by mentioning the we focus here only on the discrepancy error (7), and do not discuss the Monte-Carlo error that can't be avoided in practice. One could use the so-called multi-level Monte-Carlo method (MLMC) [10] which is a popular technique for reducing the variance. Indeed, it applies not only to first weak order integrators for SDEs but also to higher orders weak schemes, as shown in [11], where the antithetic MLMC has been introduced. Also in the context of stiff SDEs, in was shown in [1] that applying a weak second order method at the finer level of the multilevel MonteCarlo method permits to significantly improve the error constant. Finally we mention the schemes based on Markov Chain Monte-Carlo methods, see e.g. the survey [29], for which the bias error is reduced to zero, thanks to an appropriate acceptance/rejection criteria at each step.

The paper is organized as follows. In section 2, we present the basic ingredients of backward error analysis and the framework in [3] that allows us to characterise the long time behaviour of numerical integrators of ergodic SDEs. In Section 3, we prove the main result of this paper, namely sufficient conditions for a Lie-Trotter splitting scheme to capture the numerical invariant measure of nonlinear ergodic Langevin dynamics up to an arbitrary order. We also relate this characterization to geometric properties of the deterministic integrator and present in Section 4 various numerical investigations, both for linear and nonlinear problems that corroborate such theoretical findings.

\section{Preliminaries}

In Section 2.1, we discuss some important concepts related to backward error analysis for ODEs, while in Section 2.2 we discuss the standard framework of backward Kolmogorov equation and weak Taylor expansions. Finally, in Section 2.3, we recall the order conditions framework from [3] for the approximation of the invariant measure. 


\subsection{Deterministic backward error analysis}

Backward error analysis is a powerful tool for the analysis of numerical integrators for differential equations $[30,18,13]$. In particular, it is the main ingredient for the proof of the good energy conservation (without drift) of symplectic Runge-Kutta methods when applied to deterministic Hamiltonian systems over exponentially long time intervals. In our context it is useful to characterize the "generator" of the deterministic method $\Phi_{h}$ of (5). Given a consistent integrator $y_{n+1}=\Phi_{h}\left(y_{n}\right)$ for a system of ODEs

$$
\frac{d y(t)}{d t}=f(y(t))
$$

the idea of backward error analysis is to search for a modified differential equation written as a formal series in powers of the stepsize $h$,

$$
\frac{d \widetilde{y}}{d t}=f(\widetilde{y})+h f_{1}(\widetilde{y})+h^{2} f_{2}(\widetilde{y})+\ldots, \quad \widetilde{y}(0)=y_{0}
$$

such that (formally) $y_{n}=\widetilde{y}\left(t_{n}\right)$, where $t_{n}=n h$ (in the above differential equation, we omit the time variable for brevity). This means that the numerical solution can be interpreted as the exact solution of a modified ODE. The vector fields $f_{j}$ in the series can be constructed for all reasonable integrators by induction on $j[18,13]$, setting $f_{0}=f$. Notice however that this series diverges in general for nonlinear systems and needs to be truncated. Considering the truncated modified ODE at order $s$

$$
\frac{d \widetilde{y}}{d t}=f(\widetilde{y})+h f_{1}(\widetilde{y})+h^{2} f_{2}(\widetilde{y})+\ldots+h^{s} f_{s}(\widetilde{y}), \quad \widetilde{y}(0)=y_{0}
$$

we have $y_{n}=\widetilde{y}\left(t_{n}\right)+\mathcal{O}\left(h^{s+1}\right)$ for $h \rightarrow 0$ for bounded times $t_{n}=n h \leq T$. We note that the flow $\widetilde{\Phi}_{h}(y)$ of the modified differential equation (12) can be expressed as

$$
\widetilde{\Phi}_{h}=\left(\sum_{k=0}^{M} \frac{h^{k} \widetilde{\mathcal{L}}_{D}^{k}}{k !}\right) I+\mathcal{O}\left(h^{M+1}\right), \quad \widetilde{\mathcal{L}}_{D}=F_{0}+h F_{1}+h^{2} F_{2}+\ldots+h^{s} F_{s},
$$

for all $M \geq 0$, where $I$ is the identity map, $F_{j} \phi=f_{j} \cdot \nabla \phi, j=0, \ldots, s$ and $f_{0}=f$. This is a particular case $(\phi(y)=I y)$ of the general result [13, Sect. III.5.1]

$$
\phi \circ \widetilde{\Phi}_{h}=\left(\sum_{k=0}^{M} \frac{h^{k} \widetilde{\mathcal{L}}_{D}^{k}}{k !}\right) \phi+\mathcal{O}\left(h^{M+1}\right),
$$

for all smooth test function $\phi$. The constants symbolized by $\mathcal{O}$ in (13), (14), are independent of $h \rightarrow 0$ but depend on $M, s$ and $\phi .^{2}$

\subsection{Order conditions for the numerical invariant measure}

A numerical method applied to (1) is called ergodic if it has a unique invariant law $\mu^{h}$ with finite moments of any order and

$$
\lim _{N \rightarrow \infty} \frac{1}{N} \sum_{n=1}^{N} \phi\left(p_{n}, q_{n}\right)=\int_{\mathbb{R}^{d} \times \mathbb{R}^{d}} \phi(p, q) d \mu^{h}(p, q) .
$$

\footnotetext{
${ }^{2}$ For all $h$ small enough, the sum in (13) (and (14)) can be shown to converge for $M \rightarrow \infty$ in the case of analytic vector fields $f_{j}$ (and analytic test functions $\phi$ ), which permits to remove the $\mathcal{O}$ remainder.
} 
The aim of this subsection is to briefly describe the conditions that the numerical method applied to (1) should satisfy in order to approximate the invariant measure $\mu$ with an error (7) of size $\mathcal{O}\left(h^{r}\right)$. These conditions relate directly to the expansion of one-step numerical expectations in powers of $h$. In particular, let $X_{1}=\left(p_{1}, q_{1}\right), x=(p, q)$ and

$$
U(x, h)=\mathbb{E}\left(\phi\left(X_{1}\right) \mid X_{0}=x\right)
$$

We assume that it is possible to expand $U$ in powers of $h$ to obtain the following expansion

$$
U(x, h)=\phi(x)+h A_{0} \phi(x)+h^{2} A_{1} \phi(x)+h^{3} A_{2} \phi(x)+\cdots
$$

where $A_{i}, i=0,1, \cdots$ are linear differential operators with coefficients depending on the choice of the numerical integrator and depending smoothly on the Hamiltonian function $H(p, q)$ and its derivatives. For a consistent method (i.e. of weak order at least one), we have $A_{0}=\mathcal{L}$ where $\mathcal{L}$ denotes the generator of (1) given by

$$
\mathcal{L}:=p \cdot \nabla_{q}-(\nabla V(q)+\gamma p) \cdot \nabla_{p}+\beta^{-1} \gamma \Delta_{p}
$$

where $\nabla_{p}, \nabla_{q}$ denote the gradient differential operators with respect to $p$ and $q$, and $\Delta_{p}$ is the Laplacian with respect to the $p$ variable. Truncating the expansion (15) yields rigorous estimates thanks to the following result (see for instance [32]).

Example 2.1. Consider the Euler-Maruyama method for (1) defined as

$$
\begin{aligned}
q_{n+1} & =q_{n}+h p_{n}, \\
p_{n+1} & =p_{n}-h\left(\nabla V\left(q_{n}\right)+\gamma p_{n}\right)+\sqrt{2 \beta^{-1} \gamma h} \xi_{n},
\end{aligned}
$$

where $\xi_{n} \sim \mathcal{N}(0, I)$ are independent d-dimensional Gaussian random variables. A straightforward calculation yields that $A_{1}$ is a differential operator of order 4,

$$
A_{1} \phi=\frac{1}{2} \phi^{\prime \prime}(f, f)+\beta^{-1} \gamma\left(\Delta_{p} \phi\right)^{\prime} f+\frac{\beta^{-2} \gamma^{2}}{2} \Delta_{p}^{2} \phi \quad \text { with } \quad f=\left(\begin{array}{c}
-\nabla V(q)-\gamma p \\
p
\end{array}\right)
$$

where $\phi^{\prime \prime}(\cdot, \cdot)$ denotes the second differential of $\phi$ (bilinear form), and $\phi^{\prime}$ is the first derivative (linear form). We refer to [2, 35] for other examples of such weak Taylor series calculations.

We can now state the conditions which ensure that $\mu^{h}$ approximates $\mu$ to order $r$. We have the following theorem which involves the adjoint operators $A_{j}^{*}$ of the differential operators $A_{j}$ involved in (15), defined as

$$
\int_{\mathbb{R}^{2 d}} A_{j}^{*} \phi_{1}(x) \phi_{2}(x) d x=\int_{\mathbb{R}^{2 d}} \phi_{1}(x) A_{j} \phi_{2}(x) d x
$$

for all smooth test functions $\phi_{1}, \phi_{2}$ with compact support on $\mathbb{R}^{2 d}$. The following result is proved in [3].

Theorem 2.2. For the ergodic system (1), consider a consistent ergodic numerical method satisfying (15) and

$$
A_{j}^{*} \rho_{\infty}=0, \quad \text { for } \quad j=1, \cdots, r-1 .
$$


Then one obtains that the error in (7) satisfies for all test functions $\phi \in \mathcal{C}_{P}^{\infty}\left(\mathbb{R}^{2 d}, \mathbb{R}\right)$,

$$
e(\phi, h)=h^{r} \int_{0}^{\infty} \int_{\mathbb{R}^{d} \times \mathbb{R}^{d}} A_{r} u(p, q, t) \rho_{\infty}(p, q) d p d q d t+\mathcal{O}\left(h^{r+1}\right)
$$

where $\rho_{\infty}$ is the invariant measure density given in (3), $h$ is assumed small enough, and $u(x, t)$ solves the backward Kolmogorov equation

$$
\begin{aligned}
\frac{\partial u}{\partial t}(p, q, t) & =\mathcal{L} u(p, q, t), \quad t>0, p, q \in \mathbb{R}^{d}, \\
u(p, q, 0) & =\phi(p, q),
\end{aligned}
$$

and $\mathcal{L}$ is the generator of (1) defined in (16).

Theorem 2.2 generalizes a standard result by Talay [34] and Milstein [22] (see e.g. [23, Chap. 2.2,2.3] for a proof) for methods which have weak order $r$. Here, the order $r$ assumption is replaced by the weaker condition (17)). An interpretation of (17) is the following: the invariant measure $\mu$ with density $\rho_{\infty}$ of (1) is preserved up to a $\mathcal{O}\left(h^{r}\right)$ error by a single time step $X_{0} \mapsto X_{1}$ of the integrator. Precisely, if $X_{0}$ is a random variable with law $\mu$, then for all test functions $\phi \in \mathcal{C}_{P}^{\infty}\left(\mathbb{R}^{2 d}, \mathbb{R}\right)$ and $h \rightarrow 0$,

$$
\mathbb{E}\left(\phi\left(X_{1}\right)\right)=\int_{\mathbb{R}^{2 d}} \phi(x) \rho_{\infty}(x) d x+\mathcal{O}\left(h^{r}\right),
$$

where the constant symbolized by $\mathcal{O}$ is independent of $h$ assumed small enough.

Remark 2.3. Assuming that $\nabla V$ is smooth with bounded derivatives at all orders, (19) has a unique solution for all test function $\phi \in \mathcal{C}_{P}^{\infty}\left(\mathbb{R}^{2 d}, \mathbb{R}\right)$. It admits a rigorous Taylor expansion up to arbitrary order $p$ for all $h$ small enough

$$
u(x, h)=\phi(x)+h \mathcal{L} \phi(x)+\frac{h^{2}}{2} \mathcal{L}^{2} \phi(x)+\ldots+\frac{h^{p}}{p !} \mathcal{L}^{p} \phi(x)+\mathcal{O}\left(h^{p+1}\right),
$$

where the function symbolized in $\mathcal{O}$ is smooth with respect to $x$ with its all derivatives having a polynomial growth, independently of the smallness of the stepsize $h$. Notice that applying this result to the deterministic problem (12), we recover the statement (14).

\section{Accuracy of Lie-Trotter splitting methods in sampling the invariant measure}

In this section, we derive our main results. We first present in Section 3.1 sufficient order conditions for the Lie-Trotter splitting to achieve order $r$ for the invariant measure. We discuss the relation of our new characterization with geometric properties such as energy and volume conservation and show in Section 3.2 that high order for the invariant measure can be achieved without high order conservation of energy and volume.

\subsection{Order conditions to capture the invariant measure to a given order}

Using arguments from backward error analysis, we prove the following result for the accuracy of the sampling the invariant measure (3) of Langevin dynamics of the form (1). 
Theorem 3.1. Consider the ergodic system (1). Assume that $\Phi_{h}$ is a consistent method for (5) satisfying (9) with modified equation from backward error analysis (11). If the vector fields in (11) satisfy

$$
\operatorname{div}\left(f_{j} \rho_{\infty}\right)=0, \quad j=1, \ldots, r-1,
$$

then, assuming ergodicity, the Lie-Trotter splitting (4) has order $r$ of accuracy for the invariant measure. Precisely, we have for all $\phi \in \mathcal{C}_{P}^{\infty}\left(\mathbb{R}^{d}, \mathbb{R}\right)$ and $h \rightarrow 0$,

$$
e(\phi, h)=-h^{r} \int_{0}^{\infty} \int_{\mathbb{R}^{d} \times \mathbb{R}^{d}} u(p, q, t) \operatorname{div}\left(f_{r}(p, q) \rho_{\infty}(p, q)\right) d p d q d t+\mathcal{O}\left(h^{r+1}\right),
$$

where $e(\phi, h)$ is defined in (7) and $u(x, t)$ is defined in (19). The constant symbolized in $\mathcal{O}$ is independent of the stepsize $h$ assumed small enough.

Proof. Consider the method (4) and its corresponding one step expansion in powers of $h$

$$
\mathbb{E}\left(\phi\left(X_{1}\right) \mid X_{0}=x\right)=\phi(x)+h \mathcal{L} \phi(x)+\cdots+h^{r} A_{r-1} \phi(x)+h^{r+1} A_{r} \phi(x)+\mathcal{O}\left(h^{r+1}\right) .
$$

In order to prove our theorem, it is enough to show that

$$
A_{j}^{*} \rho_{\infty}=0 \quad \text { for } \quad j=1, \cdots r-1, \quad A_{r}^{*} \rho_{\infty}=\operatorname{div}\left(f_{r} \rho_{\infty}\right) .
$$

The result then follows immediately from Theorem 2.2 using the identity

$$
\int_{\mathbb{R}^{2 d}} A_{r} u(x, t) \rho_{\infty}(x) d x=-\int_{\mathbb{R}^{2 d}} u(x, t) \operatorname{div}\left(f_{r}(x) \rho_{\infty}(x)\right) d x .
$$

We now start with the calculation of $A_{j}$. In particular, given a smooth test function $\phi$ and $x \in \mathbb{R}^{2 d}$, using the semi-group property of the Markov process we have

$$
\mathbb{E}\left(\phi\left(X_{1}\right) \mid X_{0}=x\right)=\mathbb{E}\left(\phi\left(\Phi_{h} \circ \Theta_{h, n}\right)\left(X_{0}\right) \mid X_{0}=x\right)=e^{h \mathcal{L}_{S}}\left(\phi \circ \Phi_{h}\right)(x),
$$

where $e^{h \mathcal{L}_{S}} \phi$ denotes the exact flow of the Kolmogorov backward equation corresponding to (6), with generator $\mathcal{L}_{S}$ given by

$$
\mathcal{L}_{S}:=-\gamma p \cdot \nabla_{p}+\beta^{-1} \gamma \Delta_{p}
$$

We next recall the generator (13) of the truncated modified equation (12) of the integrator $\Phi_{h}$

$$
\widetilde{\mathcal{L}}_{D}=F_{0}+h F_{1}+\ldots+h^{r} F_{r},
$$

where we define the differential operators $F_{j} \phi=f_{j} \cdot \nabla \phi$ (with $f_{0}=f$ ). We have, using (23), applying Remark 2.3 for $\mathcal{L}_{S}$, and using (14) with $M=s=r$,

$$
\begin{aligned}
\mathbb{E}\left(\phi\left(X_{1}\right) \mid X_{0}=x\right) & =\left(\sum_{k=0}^{r} \frac{h^{k} \mathcal{L}_{S}^{k}}{k !}\right)\left(\sum_{k=0}^{r} \frac{h^{k} \widetilde{\mathcal{L}}_{D}^{k}}{k !}\right) \phi(x)+\mathcal{O}\left(h^{r+1}\right) \\
& =\phi(x)+h \mathcal{L} \phi(x)+\sum_{k=1}^{r} h^{k+1} A_{k} \phi(x)+\mathcal{O}\left(h^{r+1}\right)
\end{aligned}
$$


where we denote

$$
A_{k}=\sum_{j=0}^{k+1} \mathcal{L}_{S}^{k+1-j}\left(\sum_{\substack{1 \leq i \leq j \\ n_{1}+n_{2}+\cdots+n_{i}=j-i}} \frac{1}{i !(k+1-j) !} F_{n_{1}} \cdots F_{n_{i}}\right)
$$

where the second sum above is over integers $n_{1}, \ldots, n_{i} \geq 0$ and is equal to the identity $I$ when $j=0$. We obtain for all $k \geq 1$,

$$
A_{k}^{*} \rho_{\infty}=\sum_{j=0}^{k+1}\left(\sum_{\substack{1 \leq i \leq j \\ n_{1}+n_{2}+\cdots+n_{i}=j-i}} \frac{1}{i !(k+1-j) !} F_{n_{i}}^{*} \cdots F_{n_{1}}^{*}\right)\left(\mathcal{L}_{S}^{*}\right)^{k+1-j} \rho_{\infty} .
$$

Using $\mathcal{L}_{S}^{*} \rho_{\infty}=0$ and $F_{i}^{*} \rho_{\infty}=0, i=1, \cdots r-1$ we see that for $k \leq r$, the only possibly nonzero term in the above sum is obtained for $j=r+1, k=r, i=1$, i.e., $F_{r}^{*} \rho_{\infty}=\operatorname{div}\left(f_{r} \rho_{\infty}\right)$. Hence, we deduce (21) which permits to conclude the proof.

Remark 3.2. Theorem 3.1 remains valid for the analogous scheme $X_{n+1}=\Theta_{h, n} \circ \Phi_{h}\left(X_{n}\right)$ with a similar analysis.

An immediate consequence of Theorem 3.1 is the following corollary using the identity

$$
\operatorname{div}\left(f_{j} \rho_{\infty}\right)=\left(\operatorname{div}\left(f_{j}\right)-\beta f_{j} \cdot \nabla H\right) \rho_{\infty} .
$$

Corollary 3.3. Under the assumptions of Theorem 3.1, if

$$
\operatorname{div}\left(f_{j}\right)-\beta f_{j} \cdot \nabla H=0, \quad j=1, \ldots, r-1,
$$

then, assuming ergodicity, the Lie-Trotter splitting (4) has order $r$ of accuracy for the invariant measure.

In addition to the estimates on the bias (7), as discussed in [3] for Theorem 2.2, we also obtain the following exponential convergence estimate (25) which is classical for weak methods [23, Chap. 2] (see also [33, 20, 25] in the context of non-globally Lipchitz vector fields).

Remark 3.4. Under the assumptions of Theorem 2.2 or Theorem 3.1 or Corollary 3.3, there exists constants $\lambda, C, K(x)>0$ such that for all $n \geq 0$,

$$
\left|\mathbb{E}\left(\phi\left(X_{n}\right)\right)-\int_{\mathbb{R}^{d} \times \mathbb{R}^{d}} \phi(p, q) \rho(p, q) d p d q\right| \leq K(x) e^{-\lambda t_{n}}+C h^{p}
$$

where $t_{n}=n h$, the constants $C, K(x), \lambda$ are independent of $n, h$, and $h$ is assumed small enough $\left(C, K(x)\right.$ depend on $\phi$ and $K(x)$ depends on $\left.X_{0}=x\right)$.

The simplest way for condition (24) to be satisfied is to consider a method $\Phi_{h}$ of order $r$, since in this case $f_{j}=0, \quad j=1, \cdots r-1$. Indeed, in this case, standard results from backward error analysis show that the right-hand side of the modified equation (12) reads $f(\widetilde{y})+h^{r} f_{r}(y)+\ldots$ and the $f_{j}=0, j=1 \ldots, r-1$. 
Relation with results of [4] In the paper [4] the authors show that if a variational (equivalently symplectic) integrator $\Phi_{h}$ preserving the energy with order $r$ is used for solving the deterministic dynamics (5), then the Lie-Trotter splitting (4) has order $r$ of accuracy for the invariant measure. Now a symplectic method applied to a Hamiltonian system is volume preserving hence $\operatorname{div}\left(f_{j}\right)=0$ for all $j \geq 0$. Furthermore, if the method $\Phi_{h}$ has order $r$, then it also preserves energy up to order $r$, i.e. for all vectors $y$,

$$
H\left(\Phi_{h}(y)\right)=H(y)+\mathcal{O}\left(h^{r+1}\right)
$$

this condition is equivalent to $f_{j} \cdot \nabla H=0$ for all $1 \leq j \leq r-1$. If both terms in (24) are required to vanish independently, we see that we obtain order $r$ of accuracy for the invariant measure under weaker assumptions than in [4]. It is not necessary for the method to be symplectic, only volume preservation up to to order $r$ is required i.e.,

$$
\operatorname{det}\left(\frac{\partial \Phi_{h}(y)}{\partial y}\right)=1+\mathcal{O}\left(h^{r+1}\right)
$$

which is equivalent to $\operatorname{div}\left(f_{j}\right)=0$ for all $1 \leq j \leq r-1$. Note that any deterministic method $\Phi_{h}$ of order $r$ automatically satisfies (26) and (27), and thus has the desired properties. We will further see in Section 3.2 that energy and volume conservation up to order $r$ of the deterministic integrator $\Phi_{h}$ in (4) are not necessary to get order $r$ of accuracy for the invariant measure, as one can construct deterministic methods that satisfy (24) with $j=r+1$, while conserving energy and volume only up to order $r$.

Remark 3.5. In general, it is difficult to construct a numerical scheme which is simultaneously energy and volume preserving, so a choice has to be made. Indeed, already for problems with only one degree of freedom $(d=1)$, energy preserving means that the numerical method has an exact trajectory $\left\{y \in \mathbb{R}^{2 d} ; H(y)=H\left(y_{0}\right)\right\}$, and thus is a time transformation of the exact solution. Such scheme can be also volume preserving if and only if this time transformation has divergence zero, which is not satisfied by known energy preserving methods such as the AVF method [27] or energy preserving variants of collocation methods [12].

Order of accuracy in the linear case The following corollary of Theorem 3.1 states that for (non-partitioned) Runge-Kutta methods and quadratic potentials, the order of accuracy for the invariant measure of the Lie-Trotter splitting (4) is always odd, i.e. if the Runge-Kutta method has order $r$, the order of accuracy for the invariant measure of the Lie-Trotter splitting is $r+1$ if $r$ is even, and $r$ if $r$ is odd. Moreover, if the Runge-Kutta method is time-symmetric, i.e. $\Phi_{h}^{-1}=\Phi_{-h}$ then the Lie-Trotter splitting (4) samples exactly the invariant measure. We recall [13] that the method $\Phi_{h}$ is symmetric if and only if (11) has an expansion in even powers of $h$, i.e.

$$
f_{2 j+1}=0 \quad \text { for all } j \text {. }
$$

Corollary 3.6. In the linear case (i.e. $V(q)$ is quadratic), assume that $\Phi_{h}$ is a consistent (non-partitioned) Runge-Kutta method for (5). Then the order of accuracy $r$ for sampling the invariant measure of the Lie-Trotter splitting (4) is odd. Moreover, if $\Phi_{h}$ is symmetric, then the error (7) of (4) is zero for all $h$ small enough. 
Proof. Since the problem is linear, the Hamiltonian vector field in (5) has the form $f(y)=$ $A y=J^{-1} S y$ where $S$ is a constant symmetric matrix and

$$
J=\left(\begin{array}{cc}
0 & I \\
-I & 0
\end{array}\right)
$$

is the $2 d \times 2 d$ symplectic matrix. For a Runge-Kutta method applied to a linear problem, then we have the identity $\Phi(x)=R(h A) x$ where $R(z)$ is a rational function. The modified differential equation for backward error analysis of $\Phi_{h}$ then has the form (see [13, Sect. IX])

$$
\frac{d \widetilde{y}}{d t}=h^{-1} \log (R(h A)) \widetilde{y}=\left(A+\alpha_{1} h A^{2}+\alpha_{2} h^{2} A^{3}+\alpha_{3} h^{3} A^{4}+\ldots\right) \widetilde{y}
$$

with constants $\alpha_{1}, \alpha_{2}, \alpha_{3}, \ldots$ Using (28), we have $\alpha_{j}=0$ for all odd $j$ in the case of a symmetric method. Since $f_{j}(y)=\alpha_{j} A^{j+1}$, to conclude the proof using Corollary 3.3, it is sufficient to show that $\operatorname{div}\left(f_{j}\right)=f_{j} \cdot \nabla H=0$ for all even integer $j$. Indeed, for $\Phi_{h}$ of order $r$, we already have $f_{j}=0$ for all $1 \leq j<r$, while for symmetric $\Phi_{h}$, we already have $f_{j}=0$ for all odd $j$. Since $j$ is even, we have that $J A^{j+1}$ is a symmetric matrix and thus $f_{j}$ is a Hamiltonian vector field $\left(f_{j}=J^{-1} \nabla H_{j}\right.$ with $\left.H_{j}(y)=\frac{1}{2} y^{T} J A^{j+1} y\right)$, which yields $\operatorname{div}\left(f_{j}\right)=0$. In addition, using the skew-symmetry of $S A^{j+1}$, we obtain $f_{j}(y) \cdot \nabla H(y)=\alpha_{j} y^{T} S A^{j+1} y=0$. This concludes the proof.

\subsection{Energy and volume conservation are sufficient, but not necessary conditions}

Corollary 3.3 shows that energy and volume conservation up to order $r$ of the deterministic integrator $\Phi_{h}$ in (4) are sufficient conditions for the splitting method (4) to have order $r$ for the invariant measure. These are however not necessary, as shown in the following theorem which proves that applying an appropriate time transformation to a symplectic integrator of order $r$ permits to increase to order $r+1$ the accuracy for the invariant measure, while the order of accuracy for the conservation of both the energy (26) and volume (27) remains $r$. We emphasize that this time transformed numerical method is only used to illustrate a theoretical finding and we do not advocate its use in practice. Here, we use the fact that for a symplectic (possibly partitioned) Runge-Kutta method $\Phi_{h}$ applied to a (deterministic) Hamiltonian system $\dot{y}=f(y)=J^{-1} \nabla H(y)$, the modified equation for backward error analysis (11) also possesses an Hamiltonian structure of the form [13, Sect. IX.3]

$$
\frac{d \widetilde{y}}{d t}=J^{-1} \nabla H_{h}(\widetilde{y}), \quad H_{h}=H+h^{r} H_{r}+h^{r+1} H_{r+1}+\ldots,
$$

where $J$ in defined in (29), and the formal series $H_{h}$ is the modified Hamiltonian.

Theorem 3.7. Assume that $\Phi_{h}$ is a consistent symplectic integrator of order $r$ for (5) with modified Hamiltonian (31). Consider the modified integrator $\bar{\Phi}_{h}$ defined via a time transformation by

$$
\bar{\Phi}_{h}(y):=\Phi_{\alpha_{h}(y) h}(y), \quad \alpha_{h}(y):=1-h^{r} \beta H_{r}(y) .
$$

Then, assuming ergodicity, the splitting method

$$
X_{n+1}=\bar{\Phi}_{h} \circ \Theta_{h, n}\left(X_{n}\right)
$$

has order $r+1$ for the invariant measure of (1). 
Proof. We denote $f_{h}=f+h^{r} f_{r}\left(f_{r}=J^{-1} \nabla H_{r}\right)$ and $\bar{f}_{h}=f+h^{r} \bar{f}_{r}$ the vector fields (12) of backward error analysis of $\Phi_{h}$ and $\bar{\Phi}_{h}$, repectively, truncated at order $r$. Using $\alpha_{h}=1+\mathcal{O}\left(h^{r}\right)$, we have $\bar{f}_{h}=\alpha_{h} f_{h}+\mathcal{O}\left(h^{r+1}\right)$. We deduce

$$
\begin{aligned}
\operatorname{div}\left(\bar{f}_{h}\right)-\beta \bar{f}_{h} \cdot \nabla H & =\alpha_{h} \operatorname{div}\left(f_{h}\right)+\nabla \alpha_{h} \cdot f_{h}-\beta \alpha_{h} f_{h} \cdot \nabla H+\mathcal{O}\left(h^{r+1}\right) \\
& =\alpha_{h} \operatorname{div}\left(f_{h}\right)-\beta \alpha_{h}\left(\nabla H+h^{r} \nabla H_{r}\right) \cdot f_{h}+\mathcal{O}\left(h^{r+1}\right) \\
& =\mathcal{O}\left(h^{r+1}\right)
\end{aligned}
$$

where we use $\operatorname{div}\left(f_{h}\right)=0$ and an easy computation shows that $\left(\nabla H+h^{r} \nabla H_{r}\right) \cdot f_{h}=0$. This yields $\operatorname{div}\left(\bar{f}_{r}\right)-\beta \bar{f}_{r} \cdot \nabla H=0$. This shows that $\bar{\Phi}_{h}$ fulfils the assumptions of Corollary 3.3 with $r$ replaced by $r+1$ in $(24)$, which concludes the proof.

It is known for deterministic geometric integrators [13, Sect. VIII.2] that a non constant time transformation destroys in general the geometric properties of symplecticity and volume conservation. Thus, it is not surprising that the scheme (32) is energy and volume preserving up to order $r$ but not up to order $r+1$. Indeed, we have that $\operatorname{div}\left(\bar{f}_{h}\right)=$ $\operatorname{div}\left(\alpha_{h} f_{h}\right)+\mathcal{O}\left(h^{p+1}\right)=\nabla \alpha_{h} \cdot f_{h}+\mathcal{O}\left(h^{r+1}\right)=-h^{r} \beta \alpha_{h} \nabla H_{r} \cdot f_{h}+\mathcal{O}\left(h^{r+1}\right)$ is $\mathcal{O}\left(h^{r}\right)$ in general because $\nabla H_{r} \cdot f_{h} \neq 0$ (otherwise, $\Phi_{h}$ would be energy preserving up to order $r+1$ ). This implies that the idea of Theorem 3.7 cannot be applied repeatedly to achieve arbitrarily high order.

Remark 3.8. The time transformation $\alpha_{h}(y)$ in Theorem 3.7 is non-globally Lipschitz in general (see e.g. the formula (36) for the symplectic Euler method). This may lead to a non-globally Lipschitz numerical flow. One possibility to make $\alpha_{h}(y)$ Lipschitz and bounded is to consider a modification, e.g., $\left.\alpha_{h}=1-\left(1+h\left|H_{r}\right|^{2}\right)^{-1} \beta h^{r} H_{r}\right)$, which does not affect $f, \bar{f}_{r}$ in the modified equation of $\bar{\Phi}_{h}$.

\section{Numerical experiments}

In this section we illustrate the various theoretical results of this paper. We start in Section 4.1 by an example with a quadratic potential, that illustrates the results of Corollary 3.6. We then illustrate in Section 4.2 the finding of Theorem 3.1 by an example with quartic potential with various deterministic integrators $\Phi_{h}$. This section also corroborates that symplecticity is not a necessary geometric property to capture the invariant measure of Langevin dynamics with high order.

\subsection{Illustration for quadratic potential}

In Figure 1, we consider the Langevin dynamics (1) in $\mathbb{R}^{2}$ with one degree of freedom $(d=1)$ with quadratic potential $V(q)=\frac{1}{2} q^{2}$, which reduces (5) to the harmonic oscillator. We observe that (4) has the form $X_{n+1}=U_{h} X_{n}+V_{h} \xi_{n}$ where $U_{h}, V_{h}$ are constant matrices. This yields that $X_{n}$ is a Gaussian variable for all $n$, and a calculation shows that the covariance matrix $\Sigma_{h}$ of the corresponding numerical invariant measure is given by the Lyapunov equation

$$
U_{h} \Sigma_{h} U_{h}^{T}-\Sigma_{h}=V_{h} V_{h}^{T} .
$$

We compare in Figure 1 the covariance matrix $\Sigma_{h}$ with the covariance matrix $\Sigma=\beta^{-1} I$ of the exact Gaussian invariant measure and plot the 2-norm of the difference. As predicted 


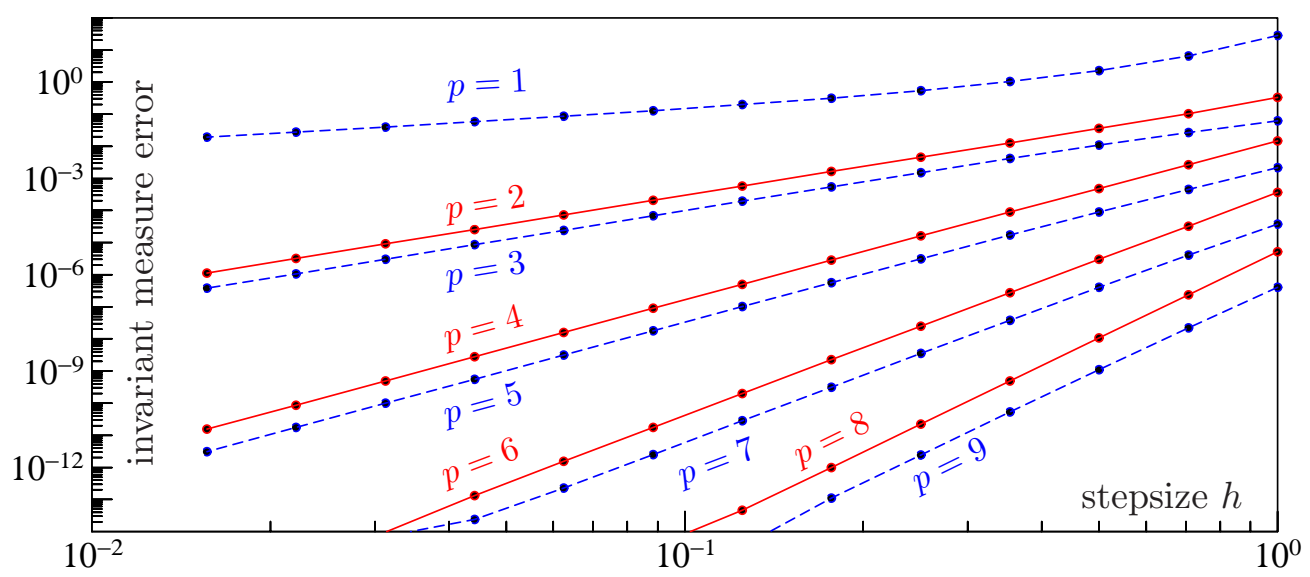

Figure 1: Accuracy of the numerical invariant measure (covariance matrix error) of the Lie-Trotter splitting for Langevin dynamics (1) in the linear case $\left(V(q)=\frac{1}{2} q^{2}\right)$. The lines corresponds to explicit deterministic integrators of orders $p=1,2,3, \ldots, 9$ (from top to bottom), respectively. The orders of accuracy for the invariant measure are always odd.

by Corollary 3.6, we observe the expected lines of with odd slopes 1, 3, 3, 5, 5, 7, 7, 9, 9 for the explicit deterministic integrators $\Phi_{h}(y)=\left(I+A+\frac{A^{2}}{2}+\ldots+\frac{A^{p}}{p !}\right) y\left(\right.$ with $\left.A=J^{-1}\right)$ of order $p$, where $p=1,2,3, \ldots, 9$, respectively.

\subsection{Illustration for quartic potential}

Although our analysis applies only to globally Lipschitz vector fields, in Figure 2, we consider the Langevin dynamics (1) with one degree of freedom with quartic potential $V(q)=\left(1-q^{2}\right)^{2}-\frac{1}{2} q$. We compare the invariant measure error $(7)$ for $\phi(p, q)=p^{2}+q^{2}$ of the Lie-Trotter splitting method (4) versus the stepsize $h$ for various choices of the deterministic integrator $\Phi_{h}$ :

- the explicit Euler method,

$$
p_{n+1}=p_{n}-h \nabla V\left(q_{n}\right), \quad q_{n+1}=q_{n}+h p_{n},
$$

- the Heun method,

$$
p_{n+1}=p_{n}-h \nabla V\left(q_{n}+\frac{h}{2} p_{n}\right), \quad q_{n+1}=q_{n}+h\left(p_{n}-\frac{h}{2} \nabla V\left(q_{n}\right)\right),
$$

- the standard symplectic Euler method,

$$
p_{n+1}=p_{n}-h \nabla V\left(q_{n}\right), \quad q_{n+1}=q_{n}+h p_{n+1},
$$

- A time transformed version of the symplectic Euler method,

$$
p_{n+1}=p_{n}-\alpha_{n} h \nabla V\left(q_{n}\right), \quad q_{n+1}=q_{n}+\alpha_{n} h p_{n+1}, \quad \alpha_{n}=1+\frac{h}{2} \beta p_{n} \cdot \nabla V\left(q_{n}\right) .
$$




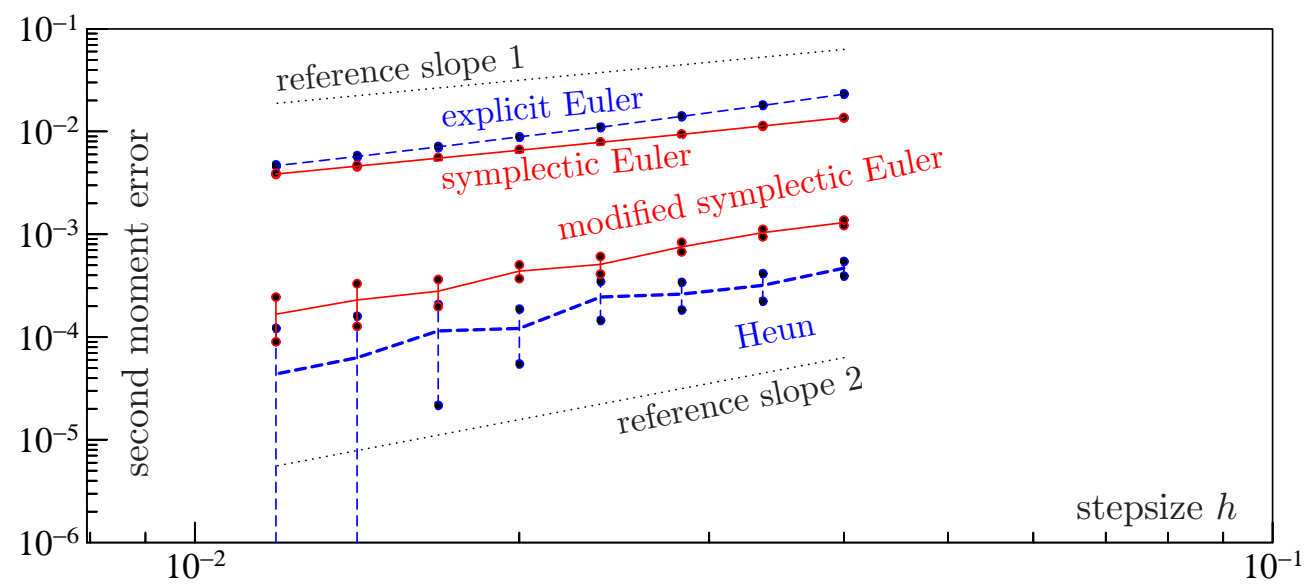

Figure 2: Invariant measure error (7) for $\phi(p, q)=p^{2}+q^{2}$ of the Lie-Trotter splitting method (4) versus the stepsize $h$ for Langevin dynamics (1) with quartic potential $V(q)=$ $\left(1-q^{2}\right)^{2}-\frac{1}{2} q(d=1)$, with $\gamma=4, \beta=2$ and initial condition $p(0)=q(0)=-1.5$. We compare various choices of the deterministic integrator $\Phi_{h}$ : explicit Euler (33), symplectic Euler (36), Heun explicit RK2 (34), and the modified symplectic Euler method (36). The curves are obtained as the averages over 10 independent long trajectories of time length $T=10^{8}$. The vertical bars measure the standard deviation intervals due to the MonteCarlo error among these 10 trajectories.

The explicit Euler method, the standard symplectic Euler method and its time transformed version have deterministic order 1 for (5), while the Heun method has deterministic order 2. In Figure 2, we plot the error (7) for $\phi(p, q)=p^{2}+q^{2}$ for the methods considered above. We consider for each method the average over 10 independent long time trajectories with length $T=10^{8}$. The vertical bars represent the standard deviation intervals due to the Monte-Carlo error among these 10 trajectories. As predicted by Corollary 3.3, the expected convergence slopes 1 and 2 can be observed in Figure 2. Precisely, the method (36) has deterministic order 1 and preserves the energy and the volume only up to order $r=1$ in (26), (27), but it has order 2 of accuracy for the invariant measure, as predicted by Theorem 3.7. We observe in Figure 2 that the order 2 schemes have an accuracy improved by a factor of about 100 compared to the order 1 methods. Again, as mentioned in Section 3.2, the time transformed symplectic Euler method (1) is only presented to illustrate Theorem 3.7 and we do not claim that it is the best method at hand to solve problem (1).

Geometric properties of symplectic and time transformed symplectic methods. In this experiment we take again the quartic potential $V(q)=\left(1-q^{2}\right)^{2}-\frac{1}{2} q$, temperature $\beta=2$ and initial condition $p(0)=q(0)=-1.5$. In Figure 3, we plot the evolution of the Hamiltonian energy (2) along time for the standard symplectic Euler method (35) and the time transformation version (36) as described in Theorem 3.7 for two values of $\gamma$.

In the top picture of Figure 3, we consider the deterministic case $(\gamma=0)$. In contrast to the standard symplectic Euler method for which no drift in the energy can be observed, we obtain a drift in the energy for the time transformed version (36) which is non symplectic. In the bottom picture, we consider the stochastic case $(\gamma=4)$ and plot sample trajectories for both methods using the same sets of generated random variables. We observe no linear 

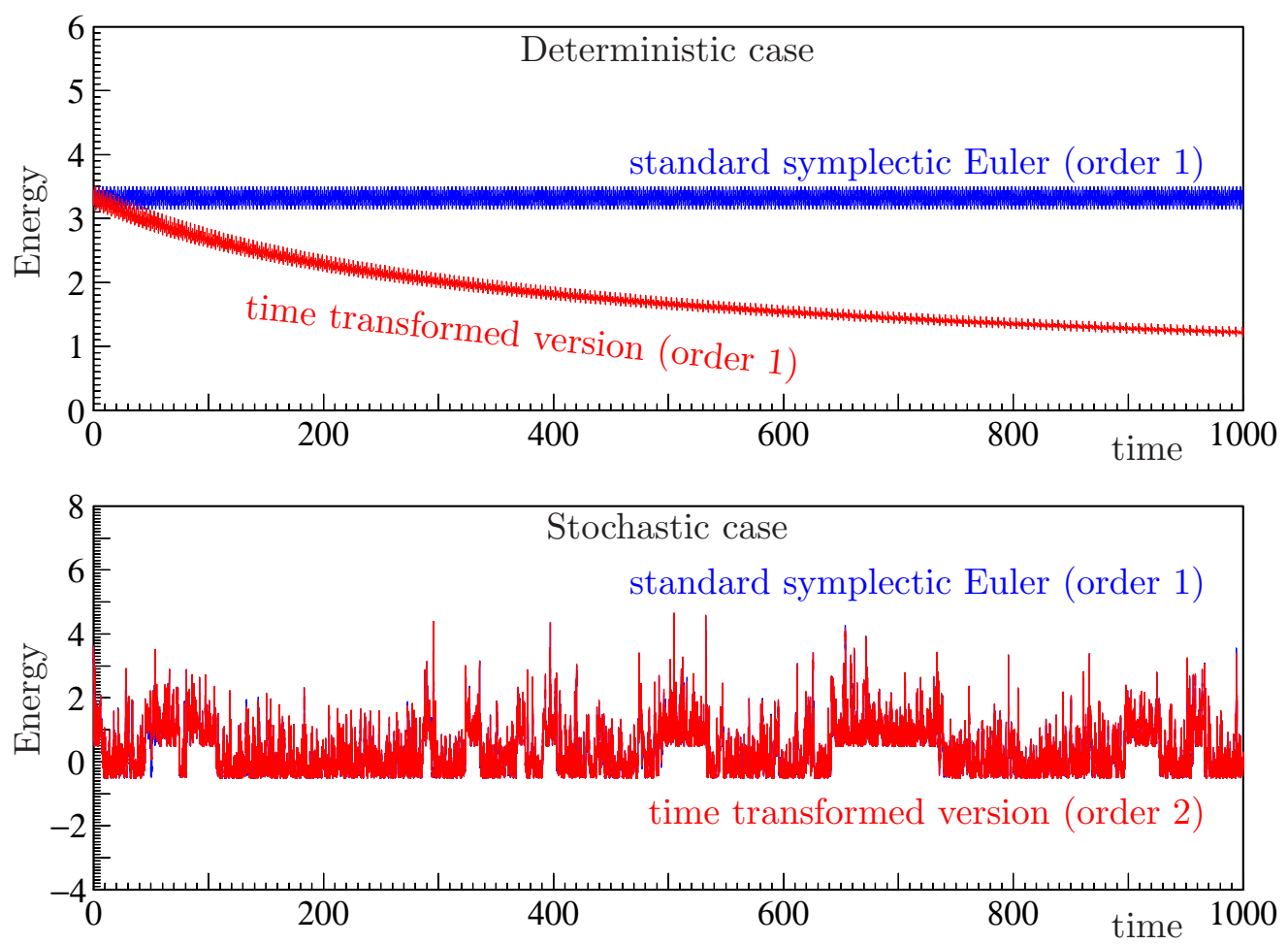

Figure 3: Evolution of the Hamiltonian energy (2) along time for the standard symplectic Euler method (35) and the time transformed version (36) (non symplectic) applied to (1) with $V(q)=\left(1-q^{2}\right)^{2}-\frac{1}{2} q$ (quartic potential). Top picture: deterministic case $(\gamma=0)$. Bottom picture: stochastic case $(\gamma=4)$. Stepsize is $h=0.02$ and $\beta=2$.

drift in the energy for both methods in this stochastic case. It can be observed in Figure 2 that the modified symplectic Euler method yields order 2 of accuracy compared to the standard version of order 1 , as predicted by Theorem 3.7. This last numerical example shows that among the standard symplectic Euler method (35) and the time transformed version (36) (applied in the Lie-Trotter splitting (4)), the most accurate scheme is not the same in the deterministic and stochastic contexts, as predicted by Theorem 3.7.

\section{Conclusion}

We have presented an analysis supported by numerical experiments of Lie-Trotter splitting methods applied to nonlinear Langevin dynamics. In particular, we obtained a new characterization of sufficient conditions for the Lie-Trotter splitting to capture the numerical invariant measure of nonlinear ergodic Langevin dynamics up to arbitrary order. While in previous results in the literature symplecticity and energy conservation (up to a given order) were required to prove high order accuracy for the numerical invariant measure, we showed that although sufficient, these conditions are not necessary. More precisely, we showed that the order of convergence of the deterministic integrator provides a lower bound for the order of convergence for the numerical invariant measure of the Lie-trotter splitting method. We illustrated this finding by constructing a first order non symplectic deterministic integrator that, despite its poor geometric properties, captures the invariant measure of Langevin dynamics to second order. 
Acknowledgements. The work of AA and GV was partially supported by the Fonds National Suisse, project No. 200021_140692. and No. 200020_144313/1, respectively.

\section{References}

[1] A. Abdulle and A. Blumenthal. Improved stabilized Multilevel Monte Carlo method for stiff stochastic differential equations. to appear in Proceedings of ENUMATH 2013, Lect. Notes Comput. Sci. Eng., 39, 2014.

[2] A. Abdulle, D. Cohen, G. Vilmart, and K. C. Zygalakis. High order weak methods for stochastic differential equations based on modified equations. SIAM J. Sci. Comput., 34(3):1800-1823, 2012.

[3] A. Abdulle, G. Vilmart, and K. C. Zygalakis. High order numerical approximation of the invariant measure of ergodic SDEs. SIAM J. Numer. Anal., 52(4):1600-1622, 2014.

[4] N. Bou-Rabee and H. Owhadi. Long-run accuracy of variational integrators in the stochastic context. SIAM Journal on Numerical Analysis, 48(1):278-297, 2010.

[5] N. Bou-Rabee and E. Vanden-Eijnden. Pathwise accuracy and ergodicity of metropolized integrators for SDEs. Communications on Pure and Applied Mathematics, 63(5):655-696, 2010.

[6] K. Burrage, I. Lenane, and G. Lythe. Numerical methods for second-order stochastic differential equations. SIAM Journal on Scientific Computing, 29(1):245-264, 2007.

[7] K. Burrage and G. Lythe. Accurate stationary densities with partitioned numerical methods for stochastic differential equations. SIAM Journal on Numerical Analysis, 47(3):1601-1618, 2009.

[8] P. Chartier, E. Hairer, and G. Vilmart. Numerical integrators based on modified differential equations. Math. Comp., 76(260):1941-1953 (electronic), 2007.

[9] A. Debussche and E. Faou. Weak backward error analysis for SDEs. SIAM J. Numer. Anal., 50(3):1735-1752, 2012.

[10] M. B. Giles. Multilevel Monte Carlo path simulation. Operations Research, 56(3):607$617,2008$.

[11] M. B. Giles and L. Szpruch. Antithetic Multilevel Monte Carlo estimation for multidimensional SDEs without Lévy area simulation. Annals of Applied Probability, 24(4):1585-1620, 2014.

[12] E. Hairer. Energy-preserving variant of collocation methods. JNAIAM, 5(1-2):73-84, 2010 .

[13] E. Hairer, C. Lubich, and G. Wanner. Geometric Numerical Integration. StructurePreserving Algorithms for Ordinary Differential Equations. Springer Series in Computational Mathematics 31. Springer-Verlag, Berlin, second edition, 2006.

[14] M. Kopec. Weak backward error analysis for Langevin process. preprint, 2013. 
[15] M. Kopec. Weak backward error analysis for overdamped Langevin processes. preprint, 2013.

[16] B. Leimkuhler and C. Matthews. Rational construction of stochastic numerical methods for molecular sampling. Appl. Math. Res. Express, pages 34-56, 2013.

[17] B. Leimkuhler, C. Matthews, and G. Stoltz. The computation of averages from equilibrium and nonequilibrium Langevin molecular dynamics. Preprint, 2013.

[18] B. Leimkuhler and S. Reich. Simulating Hamiltonian Dynamics. Cambridge Monographs on Applied and Computational Mathematics 14. Cambridge University Press, Cambridge, 2004.

[19] T. Li, A. Abdulle, and W. E. Effectiveness of implicit methods for stiff stochastic differential equations. Commun. Comput. Phys., 3(2):295-307, 2008.

[20] J. Mattingly, A. Stuart, and D. Higham. Ergodicity for SDEs and approximations: locally Lipschitz vector fields and degenerate noise. Stochastic Processes and their Applications, 101(2):185 - 232, 2002.

[21] J. C. Mattingly, A. M. Stuart, and M. V. Tretyakov. Convergence of numerical time-averaging and stationary measures via Poisson equations. SIAM Journal on Numerical Analysis, 48(2):552-577, 2010.

[22] G. Milstein. Weak approximation of solutions of systems of stochastic differential equations. Theory Probab. Appl., 30(4):750-766, 1986.

[23] G. Milstein and M. Tretyakov. Stochastic numerics for mathematical physics. Scientific Computing. Springer-Verlag, Berlin and New York, 2004.

[24] G. N. Milstein and M. V. Tretyakov. Numerical integration of stochastic differential equations with nonglobally Lipschitz coefficients. SIAM J. Numer. Anal., 43(3):11391154 (electronic), 2005.

[25] G. N. Milstein and M. V. Tretyakov. Computing ergodic limits for Langevin equations. Phys. D, 229(1):81-95, 2007.

[26] B. Oksendal. Stochastic differential equations, 6th edition. Springer-Verlag, Berlin and New York, 2005.

[27] G. R. W. Quispel and D. I. McLaren. A new class of energy-preserving numerical integration methods. Journal of Physics A: Mathematical and Theoretical, 41(4):045206, 2008 .

[28] G. O. Roberts and R. L. Tweedie. Exponential convergence of Langevin distributions and their discrete approximations. Bernoulli, 2(4):pp. 341-363, 1996.

[29] J. M. Sanz-Serna. Markov Chain Monte Carlo and Numerical Differential Equation. Lectures Notes in Mathematics 2082. Springer, Switzerland, 2014.

[30] J. M. Sanz-Serna and M. P. Calvo. Numerical Hamiltonian problems, volume 7 of Applied Mathematics and Mathematical Computation. Chapman \& Hall, London, 1994. 
[31] T. Shardlow and A. M. Stuart. A perturbation theory for ergodic markov chains and application to numerical approximations. SIAM Journal on Numerical Analysis, 37(4):1120-1137, 2000.

[32] D. Talay. Discrétisation d'une équation différentielle stochastique et calcul approché d'espérances de fonctionnelles de la solution. RAIRO Modél. Math. Anal. Numér., 20(1):141-179, 1986.

[33] D. Talay. Stochastic Hamiltonian systems: exponential convergence to the invariant measure, and discretization by the implicit Euler scheme. Markov Process. Related Fields, 8(2):163-198, 2002. Inhomogeneous random systems (Cergy-Pontoise, 2001).

[34] D. Talay and L. Tubaro. Expansion of the global error for numerical schemes solving stochastic differential equations. Stochastic Anal. Appl., 8(4):483-509 (1991), 1990.

[35] K. C. Zygalakis. On the existence and the applications of modified equations for stochastic differential equations. SIAM J. Sci. Comput., 33(1):102-130, 2011. 